\title{
Quantum Computer: Quantum Model and Reality
}

\author{
Vasil Penchev, vasildinev@gmail.com \\ Bulgarian Academy of Sciences: Institute of Philosophy and Sociology: \\ Dept. of Logical Systems and Models
}

\begin{abstract}
Any computer can create a model of reality. The hypothesis that quantum computer can generate such a model designated as quantum, which coincides with the modeled reality, is discussed. Its reasons are the theorems about the absence of "hidden variables" in quantum mechanics. The quantum modeling requires the axiom of choice. The following conclusions are deduced from the hypothesis. A quantum model unlike a classical model can coincide with reality. Reality can be interpreted as a quantum computer. The physical processes represent computations of the quantum computer. Quantum information is the real fundament of the world. The conception of quantum computer unifies physics and mathematics and thus the material and the ideal world. Quantum computer is a non-Turing machine in principle. Any quantum computing can be interpreted as an infinite classical computational process of a Turing machine. Quantum computer introduces the notion of "actually infinite computational process". The discussed hypothesis is consistent with all quantum mechanics. The conclusions address a form of neo-Pythagoreanism: Unifying the mathematical and physical, quantum computer is situated in an intermediate domain of their mutual transformations.
\end{abstract}

Key words: model, quantum computer, reality, Turing machine

\section{Eight questions:}

There are a few most essential questions about the philosophical interpretation of quantum computer. They refer to the fundamental problems in ontology and epistemology rather than philosophy of science or that of information and computation only. The contemporary development of quantum mechanics and the theory of quantum information generate them. Those eight questions can be formulated as follows:

1. Can a quantum model unlike a classical model coincide with reality?

2. Can reality be interpreted as a quantum computer?

3. Can physical processes be understood better and more generally as computations of a quantum computer?

4. Is quantum information the real fundament of the world?

5. Does the conception of quantum computer unify physics and mathematics and thus the material and the ideal world?

6. Is quantum computer a non-Turing machine in principle?

7. Can a quantum computation be interpreted as an infinite classical computational process of a Turing machine?

8. Does quantum computer introduce the notion of "actually infinite computational process"?

The possible answers of these questions will be discussed in detail bellow: 


\section{Can a quantum model unlike a classical model coincide with reality?}

There is a "central dogma" in epistemology about the irremovable difference between reality and knowledge, because of which cognition is an infinite process of the representation of reality by better and better models thus generating a well-ordered series of models in time. That well-ordered series in turn is a linear model of the development of science. Thus any model cannot coincide with reality in principle though the series of the successive models of reality converges and its limit should be an exact model coinciding with reality.

Furthermore, any classical model ever created in science corroborates that postulate: The model is always something simpler and less perfect than reality. Any quantity representing the difference between reality and any model should not be zero but, in the best case, perhaps converging to zero.

The way of the concept of 'quantum model' is crucial:

Quantum model can be defined as some subset in Hilbert space representing some part of reality.

The simplest non-trivial example of a quantum model is a wave function (i.e. a point in Hilbert space) which represents a state of a quantum system. The state can be interpreted both as a coherent superposition of all possible states of the system and as a statistical ensemble of all measurements of it.

That definition of quantum model generates a specific option for the model and reality to coincide with each other. Quantum model realizes that option being as complementary as identical to the corresponding reality in a rather paradoxical way: In comparison with this, any classical model is neither complementary nor identical to the corresponding reality. One can say that the cost of that coincidence is quantum complementarity since it is a necessity condition.

Infinity in turn can be considered as another condition of coincidence of a model and reality: Indeed Hilbert space is infinitely dimensional, but even the finitely dimensional subspaces of it involve infinity by virtue of the "Banach - Tarski paradox" (Banach, Tarski 1924): In fact only an infinite set can be divided into two parts such that three one-to-one mappings exist between any two of them accordingly. The opposite is also true: that division of a set means that it is infinite.

\section{Is reality interpretable as a quantum computer?}

In order to answer that question, one should define what a quantum computer is: Utilizing the above coincidence of a quantum model and reality, a quantum computer can be defined as what processes a quantum model and therefore reality itself. Furthermore, any physical quantity or its change both definable as a self-adjoint operator in Hilbert space in quantum mechanics can be represented as a corresponding computation of a quantum computer.

Quantum computer can be also defined as that computer, all possible states of which are coherent states of a quantum system. Thus all of them can be described by corresponding wave functions. As all wave functions are points in Hilbert space, the quantum computer can be equivalently defined as a series of successive transformations (operators) of Hilbert space into itself: Thus a single quantum computation is any of those transformations (operators). One can easily show that the above definition of quantum computer can be interpreted as the corresponding generalization of a Turing machine to a quantum one. Indeed the quantum 
computer can be equivalently defined as a quantum generalization of a Turing machine, in which all bits ("cells") are replaced by qubits:

'Qubit' is: " $\alpha|0\rangle+\beta|1\rangle$ " where " $\alpha, \beta$ " are two complex numbers such that $"|\alpha|^{2}+|\beta|^{2}=1$ ", and " $|0\rangle,|1\rangle$ " are any two orthonormal vectors (e.g. the orthonormal bases of any two subspaces) in any vector space (e.g. Hilbert space, Euclidean space, etc.)

Hilbert space can be easily and equivalently represented as a "tape" of qubits as follows: Given any point in (complex) Hilbert space as a vector " $\left\{C_{1}, C_{2}, \ldots C_{n}, C_{n+1}, \ldots\right\}$ ", one can replace any successive couple of its components such as " $\left(\left\{C_{1}, C_{2}\right\},\left\{C_{2}, C_{3}\right\}, \ldots\right.$ $\left.\left\{C_{n-1}, C_{n}\right\} \ldots\right)$ " with a single corresponding qubit $\left\{Q_{1}, Q_{2}, \ldots, Q_{n}, Q_{n+1}, \ldots\right\}$ such that: “ $\alpha_{n}=\frac{C_{n}}{(+) \sqrt{\left|C_{n}\right|^{2}+\left|C_{n+1}\right|^{2}}}$;

$\beta_{n}=\frac{C_{n+1}}{(+) \sqrt{\left|C_{n}\right|^{2}+\left|C_{n+1}\right|^{\prime}}}$ " if " $C_{n}, C_{n+1}$ " are not both " 0 ". However if both are " 0 " one needs to add conventionally the center " $\left(\alpha_{n}=0, \beta_{n}=0\right)$ " to conserve the mapping of Hilbert space and an infinite qubit tape to be one-to-one.

This interpretation allows of interpreting any physical process as a quantum computation:

Indeed the quantum computer can be equivalently represented by a quantum Turing machine. A quantum Turing machine is equivalent to Hilbert space. Quantum mechanics states that any physical state or its change is a self-adjoint operator in Hilbert space as any physical system can be considered as a quantum one. Consequently all physical process can be interpreted as the calculation of a single computer and thus the universe being as it.

\section{Can physical processes be understood better and more generally as computations of quantum computer?}

Yes, they can, as being computations, they should share a common informational fundament, which is hidden from any other viewpoint to the physical processes. However, that fundament cannot be that of the information defined classically (e.g. in Shannon) but it should be generalized as a new kind of information: quantum information.

Then any wave function being a point in Hilbert space can be reckoned as a concrete value of quantum information. Indeed any wave function can be represented as an ordered series of qubits enumerated by the positive integers: Just as an ordering of bits can represent a value of classical information, that series of qubits, equivalent to a wave function represents a value of quantum information. One can think of the qubits of the series as a special kind of digits: infinite digits. As a binary digit can accept two values, that infinite digit should accept infinite values.

Furthermore, any physical quantity can be interpreted as a quantum computation: Indeed any physical quantity according to quantum mechanics corresponds to a self-adjoint operator and thus to a certain change of a wave function. Any wave function represents a state of a quantum computer. Consequently, any physical quantity should correspond to a quantum computation defined as the change the state of a quantum computer.

In fact all physical processes can be considered as informational ones. This can be demonstrated as follows: Quantum mechanics is the universal doctrine about the physical world and any physical process can be interpreted as a quantum one. Any quantum process is informational in terms of a generalized kind of information: quantum information. 
Consequently, all physical processes are informational in the above sense.

\section{Is quantum information the real fundament of the world?}

The answer should be positive again: All physical states in the world are wave functions and thus they are different values of quantum information. All physical quantities in the world are a certain kind of changes of wave functions and thus of quantum information. Consequently, one can certainly state that the physical world consists of only quantum information: It is the substance of the physical world, its 'matter'.

The way of how quantum information is defined is crucial. However "quantum information" can be defined in a few equivalent ways:

- As the information in Hilbert space.

- As the information measured in quantum bits (qubits).

- As the information concerning infinite sets.

- As the information in a wave function.

- As the information in any quantum state or process.

- Etcetera.

Quantum information can be compared with classical information in a few features:

- If classical information refers only to finite sets, quantum information is defined immediately only to infinite sets.

- Quantum information can be discussed both as the counterpart of classical information to the infinite sets and as the generalization of classical information including both the finite and infinite sets.

- The latter requires the axiom of choice, the former does not.

The interrelations between the concepts of information and choice are very close. Indeed information either classical or quantum can be defined as the quantity of the units of choice in an entity:

- The unit of classical information is a choice between a given finite set of equiprobable alternatives, e.g. a bit is a choice among two equiprobable alternatives usually designated by " 0 " and " 1 ".

- Analogically, the unit of quantum information, a qubit is a choice among infinite equiprobable alternatives therefore requiring the axiom of choice in general.

\section{Does the conception of quantum computer unify physics and mathematics and thus the material and the ideal world?}

As information is a dimensionless quantity equally well referring both to a physical entity and to a mathematical class, it can serve as a "bridge" between physics and mathematics and thus between the material and ideal world. In fact quantum information being a generalized kind of information is just what allows of the physical and mathematical to be considered as two interpretations of the underlying quantum information.

This allows of interpreting physics as a branch of mathematics: Classical physics and science distinguish fundamentally the mathematically models from the modeled reality and thus there is a "strong interface" between mathematics and physics. However, quantum mechanics, erasing that boundary, therefore understands physics newly: as a special branch 
of mathematics, in which two kinds of models coincide in principle: Then reality (and therefore physics) turns out to be defined within mathematics as the one kind of models.

One can build a reliable quantum pathway between physics and mathematics. The bridge between physics and mathematics, built by quantum mechanics necessarily utilizes the concept of infinity.

Mathematics has introduced that concept in its fundament since the end of the $19^{\text {th }}$ century by set theory. Quantum mechanics is the only experimental science about reality, which has forced also to involve infinity in its ground to describe uniformly quantum (leap-like) and smooth (continuous) motion since the first half of the $20^{\text {th }}$ century.

The mathematical foundation of the physical world needs a reinterpretation. Quantum mechanics shows how the physical world can be grounded on a mathematical theory involving infinity, that of Hilbert space. It is infinitely dimensional in general and thus infinity is involved. However infinity is implicitly included in any finitely $(\mathrm{N})$ dimensional subspace of Hilbert space by dint of its basis: " $e^{i n \omega}, n=1, \ldots, N$ ".

\section{Is quantum computer a non-Turing machine in principle?}

The answer should be positive. Indeed the concept of infinity allows of distinguishing a quantum computer from a Turing machine. A Turing machine should end its computation in a finite number of steps and in a finite time as any step is accomplished in a finite but nonzero time. A quantum computer can finish making an infinite number of steps necessity of a Turing machine as a whole by a leap in a finite time even zero theoretically.

A Turing machine can be generalized to a quantum Turing machine as follows.

First one can restrict the set of Turing machines to that subset of them, which have finished the work with a result. That subset is equivalent to the set of all finite positive integers (e.g. represented binary). For a quantum computer not to be equivalent to any Turing machine, it should not belong to the above set, which is enumerable.

The next step is a relevant definition of a quantum Turing machine. It being equivalent to a quantum computer can be defined as a Turing machine, in which all bits are replaced by qubits as above. Any qubit is a choice among an uncountable set such as the points of a 3D unit sphere. Consequently, a quantum computer is not a Turing machine in general as all its possible states are an uncountable set unlike a Turing machine.

Furthermore, even a single quantum computation should be represented as infinite on a Turing machine. However a quantum computation represented thus is both infinite and converging: that is a converging series of Turing machines finished the work with a partial result. So, all possible states of a Turing machine and those of a quantum computer are related to each other as a set of rational numbers to that of irrational ones within any finite interval or area. Consequently, a quantum computation on a Turing machine will require infinitely many steps and cannot ever end.

\section{Can a quantum computation be interpreted as an infinite classical computational process of a Turing machine?}

Consequently a quantum computation can be interpreted thus. However, a quantum computer can accomplish that computation ending with a finite result in a finite time making a quantum jump to the limit of the process. While a Turing machine cannot do that leap and cannot stop ever yielding the result. 
The quantum computation needs the axiom of choice. Any quantum computation in general makes a jump and thus it chooses the result in a non-constructive way therefore requiring the axiom of choice. Quantum computation is grounded both on infinity and the axiom of choice. However, the quantum computation is invariant to the axiom of choice in a sense for the result is single and necessary and thus no choice is made. Only the non-constructiveness of the quantum computation is only what remains from it.

Involving the axiom of choice a series of counterintuitive conclusions is implied: For exammple this implies the equivalence of a single qubit and arbitrarily many qubits. Indeed, as an infinite set has the same power as the set of arbitrarily many sets, each of the same power, the choice of a point among a qubit is equivalent to the choice of arbitrarily many points, each from a qubit. The same can be deduced from the so-called Banach-Tarski (1924) paradox for a qubit is isomorphic to the choice of a point among those of a 3D sphere.

Quantum computation can be also understood as the process converging. Being infinite, a quantum computation must be converging to a finite limit. This is guaranteed as any qubit is limited and the axiom of choice always allows of reordering any series to a monotonic one. (Any limited monotonic series has necessarily a limit.) However, there are in general two monotonic reordering for any series: either ascending or descending implying two complementary limits as any reordering excludes the other.

\section{Does quantum computer introduce the notion of "actually infinite computational process"?}

In fact quantum computer requires for the computational processes to be actually infinite in general: Indeed the existing of a limit of a series means that it should be taken as completed whole (the limit is possible not to belong to the sequence at all). Thus the concept of actual infinity involves implicitly a pair of an infinite series and a limit, to which the series should converge in principle.

The limit of an infinite computation can be interpreted as a quantum leap. Rather paradoxically, the concept of actual infinity unlike that of potential infinity implies Skolem's relativity (1922) of the sets by mediation of the axiom of choice. Thus the limit of an infinite computation can be considered as the finite and complementary, quantum counterpart of the corresponding infinite series of the computation. Max Born's "interpretation" (1926) of quantum mechanics implies the same statement and thus the infinite series and its limit are the two complementary representations of a quantum state interpreted as a quantum computation.

Furthermore the limit of an infinite computation can be interpreted also as actual infinity. In fact the concept of 'limit' of an infinite series has already introduced that of 'actual infinity' as: There is an infinite set, that of the members of the series in question. The set must be considered as a completed whole in the limit of the series, which can be as finite as infinite, as a member of the series, as not such a one. Thus the limit represents the infinite series as a singularity.

Consequently, the quantum computation as a whole can be considered as an infinite computation. Quantum computation can be represented as an infinite series of partial results or "Turing machines" necessarily converging to a limit. Consequently, it is an infinite computation if it is modeled on a set of Turing machines. The transition to the limit is always a leap from any partial result: Thus that jump being just quantum in fact cannot be 
accomplished by any Turing machine.

\section{Conclusions:}

- A quantum model unlike a classical model can coincide with reality.

- Reality can be interpreted as a quantum computer.

- The physical processes represent computations of the quantum computer.

- Quantum information is the real fundament of the world.

- The conception of quantum computer unifies physics and mathematics and thus the material and the ideal world.

- Quantum computer is a non-Turing machine in principle.

- Any quantum computing can be interpreted as an infinite classical computational process of a Turing machine.

- Quantum computer introduces the notion of "actually infinite computational process".

\section{References:}

Banach S, Tarski A (1924) "Sur la decomposition des ensembles de points en parties respectivement congruentes," Fundamenta Mathematicae 6 (1): 244-277.

Born M (1926a) "Zur Quantenmechanik der Stoßvorgänge," Zeitschrift für Physik 37 (12): 863-867.

Born M (1926b) “Quantenmechanik der Stoßvorgänge," Zeitschrift für Physik 38 (11-12): 803-827

Kochen S, Specker, E (1968) “The problem of hidden variables in quantum mechanics," Journal of Mathematics and Mechanics 17 (1): 59-87

Neumann J von (1932) Mathematische Grundlagen der Quantenmechanik. Springer, Berlin

Skolem T (1922) "Einige Bemerkungen zur axiomatischen Begründung der Mengenlehre. Matematikerkongressen i Helsingrofs den 4-7 Juli 1922," Den femte skandinaviska

matematikerkongressen, Redogörelse, Helsinki: Akademiska-Bokhandeln, 1923, pp. 217-232 